\title{
Steady axisymmetric vortex flows with swirl and shear
}

\author{
ALAN R. ELCRAT ${ }^{1}$, BENGT FORNBER G $^{2}$ \\ AND KENNETH G. MILLER ${ }^{1}$ \\ ${ }^{1}$ Department of Mathematics, Wichita State University, Wichita, KS 67260, USA \\ ${ }^{2}$ Department of Applied Mathematics, University of Colorado, Boulder, CO 80309, USA
}

(Received 28 December 2007 and in revised form 2 July 2008)

A general procedure is presented for computing axisymmetric swirling vortices which are steady with respect to an inviscid flow that is either uniform at infinity or includes shear. We consider cases both with and without a spherical obstacle. Choices of numerical parameters are given which yield vortex rings with swirl, attached vortices with swirl analogous to spherical vortices found by Moffatt, tubes of vorticity extending to infinity and Beltrami flows. When there is a spherical obstacle we have found multiple solutions for each set of parameters. Flows are found by numerically solving the Bragg-Hawthorne equation using a non-Newton-based iterative procedure which is robust in its dependence on an initial guess.

\section{Introduction}

Steady axisymmetric vortices with swirl as solutions of the Euler equations are of interest for several reasons. Unlike axisymmetric flows without swirl the vortex stretching terms do not vanish and the helical-like streamlines inside the vortex are, in general, ergodic with associated mixing properties. Nevertheless these threedimensional flows can be obtained as solutions to elliptic boundary value problems in two variables. We obtain families of inviscid flows in which an axisymmetric vortex with swirl is embedded in an external flow. The external flow may be irrotational flow which is uniform at infinity or it may include shear. We will consider both flows past a sphere and flows in which the vortex is the only disturbance to the flow at infinity. The solutions obtained can be related to explicit solutions of classical interest such as Hill's vortex and its generalization to flows with swirl found by Moffatt (1969) and Hicks (1899).

A number of studies have indicated that under appropriate conditions inviscid flows provide accurate models for physical flows with vorticity. The family of inviscid vortex rings, without swirl or shear, described in Norbury (1973) have been shown to compare well with numerical simulations of the Navier-Stokes equations in Wakelin \& Riley (1997), and with physically generated flows in Mohseni \& Gharib (1998) and Linden \& Turner (2001). Moreover, asymptotic expansions for NavierStokes solutions at large Reynolds numbers given in Fukumoto (2002) indicate the relevance of these inviscid solutions to Navier-Stokes solutions. The vortex rings found here when there is no obstacle can be regarded as an extension of the Norbury family to include swirl and shear. As in Norbury (1973), the vorticity profile used in the present study, (1.3) below, is that prescribed by the Prandtl-Batchelor model, Batchelor $(1956 a, b)$, without vortex sheets. There have also been several studies of the 
significance of the Prandtl-Batchelor model to flows past a bluff body; see Fornberg (1993) for an overview. That the Prandtl-Batchelor model could include flows with swirl was shown in Batchelor (1956a), a simpler proof due to Chernyshenko being given in Wu, Ma \& Zhou (2006). Therefore, it is not unreasonable to anticipate that inviscid flows likewise provide good models when swirl and shear are included, although we are not aware of any direct verifications of this.

If $r, \theta$ and $z$ are cylindrical coordinates, a flow is axisymmetric if the velocity field is independent of $\theta$. It follows that there exists a stream function $\psi$ such that

$$
v_{r}=-\frac{1}{r} \frac{\partial \psi}{\partial z}, \quad v_{z}=\frac{1}{r} \frac{\partial \psi}{\partial r},
$$

and that the components of the vorticity are given by

$$
\omega_{r}=-\frac{1}{r} \frac{\partial C}{\partial z}, \quad \omega_{z}=\frac{1}{r} \frac{\partial C}{\partial r}, \quad \omega_{\theta}=-\frac{1}{r} \mathrm{~L} \psi
$$

where $C=r v_{\theta}$ is the swirl and

$$
\mathrm{L} \psi=\frac{\partial^{2} \psi}{\partial z^{2}}+\frac{\partial^{2} \psi}{\partial r^{2}}-\frac{1}{r} \frac{\partial \psi}{\partial r} .
$$

It follows from the steady Euler equations (see Batchelor 1967, pp. 543-544) that $C=C(\psi)$ and that steady axisymmetric flows with swirl can be found by solving the Bragg-Hawthorne equation

$$
\mathrm{L} \psi=r^{2} f(\psi)-C(\psi) \frac{\mathrm{d} C}{\mathrm{~d} \psi} .
$$

The profile functions $f$ and $C$ can a priori be arbitrary, but a reasonable theory has only been given when they satisfy some restrictions. Two of us, Elcrat \& Miller (2003), have given fairly general conditions on $f$ and $h=-C C^{\prime}$ that lead to existence of solutions analogous to those found here numerically. We restrict ourselves in the computations in this paper to

$$
\left.\begin{array}{l}
f(\psi)=\sigma+\omega H(\alpha-\psi), \\
C(\psi)=\lambda(\alpha-\psi)_{+},
\end{array}\right\}
$$

where $H$ is the Heaviside function and $s_{+}=s$ if $s \geqslant 0$, and 0 if $s<0$. The constant $\alpha$ is the value of the stream function $\psi$ on the boundary of the vortex, $\sigma$ is the shear parameter for the background flow, and $\omega$ and $\lambda$ will be referred to as the vorticity and swirl parameters respectively. More general $f$ and $h$ could be dealt with using our methods, but we use these in order to fix ideas and more easily make comparisons with previous work.

By (1.1)-(1.3) the vorticity of the flows we are considering satisfies

$$
\left.\begin{array}{ll}
\boldsymbol{\omega}=-\lambda \boldsymbol{v}-r(\sigma+\omega) \boldsymbol{e}_{\theta} & \text { inside } D, \\
\boldsymbol{\omega}=-r \sigma \boldsymbol{e}_{\theta} & \text { outside } D,
\end{array}\right\}
$$

where $D=\{\psi<\alpha\}$ is the vortex region and $\boldsymbol{e}_{\theta}$ is the unit vector in the $\theta$-direction. So there is a jump in the vorticity at the boundary of the vortex region.

In Elcrat, Fornberg \& Miller (2001) solutions for flow past a sphere were computed when there is no shear and no swirl and the present algorithm is an extension of the one used in that previous work. Equation (1.2) is solved using iterations patterned 
after

$$
\mathrm{L} \psi_{n+1}=r^{2} f\left(\psi_{n}\right)+h\left(\psi_{n}\right) .
$$

However, a key step in our previous work, Elcrat et al. (2000) and Elcrat et al. (2001), was that, in order to stabilize the algorithm, $\omega$ must be adjusted to a new value $\omega_{n}$ at each iterative step so as to fix either the area of the vortex (for the two-dimensional problem) or the first moment of the vortex cross-section with respect to the $z$-axis in the meridional plane (for the axisymmetric problem). We employ the same procedure again here, as described below. As in our previous work the flows are naturally divided into classes according to whether $\alpha<0$ (vortex rings), $\alpha=0$ (attached vortices, e.g. Hill's vortex) and $\alpha>0$ (vortex tubes extending to infinity.)

When there is no obstacle, vortex rings with swirl were computed in Eydeland \& Turkington (1988) using an approach based on variational methods developed in Turkington (1989). A modification of that approach was used in Lifschitz, Suters \& Beale (1996) to study the onset of instability of rings with swirl.

There are two cases for which the solutions we have found numerically can also be given analytically. For non-swirling shear flow without an obstacle there is a generalization of Hill's spherical vortex in which the vortex boundary is a prolate spheroid. For swirling flow, without shear, there is a generalization of Moffatt's spherical vortex to flow past a sphere, where the vortex boundary is a sphere concentric with the spherical obstacle. The analytic solutions in both of these cases are discussed below.

\section{Numerical method}

The vortices being considered are embedded in a background flow which may include shear. This background flow is a perturbation of flow with uniform velocity unity at infinity by shear flow in the axial direction. Specifically, in cylindrical coordinates the background flow velocity satisfies $\boldsymbol{v} \sim\left(1+\frac{1}{2} \sigma r^{2}\right) \boldsymbol{e}_{z}$, as $r^{2}+z^{2}$ goes to infinity, where $\sigma \geqslant 0$ is constant. If $\sigma=0$ there is no shear. Let $\psi_{0}$ denote the stream function, vanishing on the axis of symmetry, for this background flow. Then $\psi_{0}$ satisfies

$$
\left.\begin{array}{ll}
\mathrm{L} \psi_{0}=\sigma r^{2} & \text { for } r^{2}+z^{2}>a^{2}, \\
\psi_{0}=0 & \text { if } r^{2}+z^{2}=a^{2} \text { or } r=0, \\
\psi_{0} \sim \frac{r^{2}}{2}+\frac{\sigma r^{4}}{8} & \text { as } r^{2}+z^{2} \rightarrow \infty
\end{array}\right\}
$$

where $a$ is the radius of the spherical obstacle. The function $\psi_{0}$ can be given analytically (see Rubel 1986) using spherical coordinates $\rho$ and $\phi, z=\rho \cos \phi$, $r=\rho \sin \phi$, as follows:

$$
\psi_{0}=\frac{1}{2} r^{2}\left(1-\frac{a^{3}}{\rho^{3}}\right)+\frac{\sigma \rho^{4}}{8} \sin ^{2} \phi\left[\left(1-\frac{a^{3}}{\rho^{3}}\right) \sin ^{2} \phi-\frac{4}{5}\left(\frac{a^{5}}{\rho^{5}}-\frac{a^{7}}{\rho^{7}}\right)\right] .
$$

Setting $u=\psi-\psi_{0}$, it follows from (1.2) and (1.3) that we are looking for solutions $u$ to

$$
\mathrm{L} u= \begin{cases}r^{2} \omega+\lambda^{2}\left(\alpha-\left(u+\psi_{0}\right)\right), & \psi<\alpha \\ 0, & \psi>\alpha\end{cases}
$$


Our basic iterative scheme for solving this nonlinear equation is

$$
\left(\mathrm{L}+\lambda^{2} I_{n}\right) u_{n+1}=\left(r^{2} \omega+\lambda^{2}\left(\alpha-\psi_{0}\right)\right) I_{n}
$$

where $I_{n}$ is the characteristic function of the set $D_{n}=\left\{\psi_{n}<\alpha\right\}$. We wish to solve (2.3) using finite differences and then iterate until the set of grid points where $\psi_{n+1}<\alpha$ is identical with the set of grid points where $\psi_{n}<\alpha$. However, as in Elcrat et al. (2000) and Elcrat et al. (2001), this basic iterative scheme is unstable: the sets $D_{n}$ may increase without bound unless there is some constraint on the size of $D_{n}$. So we modify the iterative scheme by requiring that a geometric quantity, either the area $A$ of $D_{n}$ or the first moment $M$ of $D_{n}$ with respect to the axis of symmetry, have a prescribed value. The value of one of the three parameters $\omega, \lambda$ or $\alpha$ is then adjusted in an inner iteration so as to achieve this. Thus there are four free parameters to the general problem: the shear parameter $\sigma$, a geometric parameter $A$ or $M$, and two of the three parameters $\omega, \lambda$ and $\alpha$, the remaining one of these parameters being determined as part of the solution. We generally use $M$ as the geometric parameter, since in the case $\lambda=0$, the circulation around the vortex, $k=(\omega+\sigma) M$, is determined immediately from the parameters. Which of the three parameters $\omega, \lambda$ and $\alpha$ is allowed to vary depends on the particular flow characteristics being sought. The radius $a$ of the sphere could be an alternative parameter; we have generally normalized the problem by taking $a=1$ when there is a spherical obstacle.

For flow past a sphere of radius $a$, the stream function is defined on the region $r^{2}+$ $z^{2}>a^{2}$ in the meridional half-plane $r>0$. In order to use a rectangular computational grid, we map this region onto the strip $-\pi<\xi<0, \log a<\eta<\infty$, as in Elcrat et al. (2001), via the transformation $\xi+\mathrm{i} \eta=\mathrm{i} \log (z+\mathrm{i} r)$. We then truncate the strip at $\eta=H$, for some $H$, which corresponds to truncating in the physical domain at some large sphere of radius $R=\mathrm{e}^{H}$. Typically we take $H=\pi$. The differential equation (2.3) for $u=u_{n+1}$ transforms to

$$
\frac{\partial^{2} u}{\partial \xi^{2}}+\frac{\partial^{2} u}{\partial \eta^{2}}-(\cot \xi) \frac{\partial u}{\partial \xi}-\frac{\partial u}{\partial \eta}+\mathrm{e}^{2 \eta} \lambda^{2} I_{n} u=\left(\omega e^{4 \eta} \sin ^{2} \xi+\mathrm{e}^{2 \eta} \lambda^{2}\left(\alpha-\psi_{0}\right)\right) I_{n} .
$$

The boundary condition on three sides of the computational rectangle is $u=0$. As explained in Elcrat et al. (2001), a reasonable numerical boundary condition to impose on the upper boundary $\eta=\log R$ is the Robin condition,

$$
\frac{\partial u}{\partial \eta}+2 u=0
$$

We discretize (2.4) using the standard stencil for a uniform $N_{1}$ by $N_{2}$ grid on the computational rectangle. We use the sparse matrix functionality of MATLAB to solve the resulting $N_{1} N_{2}$ by $N_{1} N_{2}$ linear system at each iteration step. We use the same method as in Elcrat et al. (2001) to compute the moment of $M$ of the set $D_{n}$.

This procedure can be modified in the case when there is no spherical obstacle. In that case the conformal mapping given above maps onto a doubly infinite strip which is then truncated at both ends, i.e. truncating in the physical domain at a small sphere as well as a large sphere.

An alternative approach would be to use a uniform rectangular grid in spherical coordinates $\rho$ and $\phi, z=\rho \cos \phi, r=\rho \sin \phi$. We tested using this approach, specifically in the case of no obstacle, for which this approach seems most likely to offer some advantage since no truncation near 0 would be required. In the case of no obstacle and $\alpha=0$ there are explicit solutions, namely Hill's spherical vortex and Moffatt's generalization with swirl, which can be used to test the accuracy of the 
numerical solutions. We found that for a fixed number of grid points, the procedure using the logarithmic transformation is more accurate than that using spherical coordinates directly, except very close to the origin. The reason for greater accuracy using the logarithmic transformation is that it concentrates more grid points in the region where the vortex occurs.

The computational algorithm that we have used and developed here, and which was initiated previously in Elcrat et al. (2000) and Elcrat et al. (2001), is very robust. Although it is based on the simple idea of successive iteration, it converges even for very crude initial guesses. We believe that this is due to a smoothing property of the linear elliptic operators involved: if a highly disconnected set is used as the set $D_{0}$ in (2.3), after one iteration the new set is connected.

There are a few cases where our method has come up against barriers in continuing solutions along branches; one is mentioned in the following section. We believe this is due to numerical instability and that an algorithm with accelerated convergence is required. The obvious choice of Newton's method does not admit straightforward implementation because of the discontinuities on the right-hand side of (2.2). We leave the resolution of these continuations to a future work.

Three-dimensional streamline plots are given in the next section for several flows. The following procedure was used to compute these streamlines based on the computed stream function $\psi$. First a numerical arclength parametrization of a streamline $\psi(z, r)=c$ in the meridional plane is found. The gradient $\nabla \psi$ is also computed numerically. By numerically integrating

$$
\mathrm{d} t=\frac{r}{\|\nabla \psi\|} \mathrm{d} s
$$

the time taken to go from a fixed initial point on the streamline to an arbitrary point is determined. Then the differential equation

$$
\frac{\mathrm{d} \theta}{\mathrm{d} t}=\frac{\lambda(\alpha-\psi)}{r^{2}}
$$

is solved numerically to determine the values of $\theta$ at those time values corresponding to equally spaced points along the meridional-plane streamline. For a closed meridionalplane streamline it is only necessary to do this calculation for one traverse of that streamline, since the incremental change in $\theta$ for subsequent loops around the toroidal surface $\psi(z, r)=c$ is the same as for the first loop. (By one 'loop' of a three-dimensional streamline we mean that the values of $r$ and $z$ make one traverse of the meridional plane streamline $\psi(z, r)=c$. In the plots we usually follow a single streamline for about 20 loops around its toroidal surface.) We have found this method to be more reliable, more accurate and more computationally efficient than solving numerically the system of three differential equations determined by the velocity field.

\section{Results}

We first consider vortex rings $(\alpha<0)$ and the limiting case $\alpha=0$, which we refer to as attached vortices, discussing cases with a spherical obstacle before considering the case without an obstacle. Vortex tubes $(\alpha>0)$ are discussed last.

\subsection{Non-swirling flows with shear}

For non-swirling flow, without shear, past a sphere, we described in Elcrat et al. (2001) four different families of solutions, parametrized by $\alpha$ and the moment $M$ of 
(a)

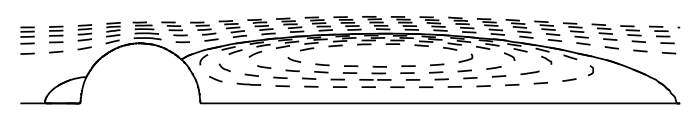

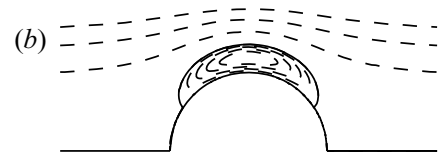
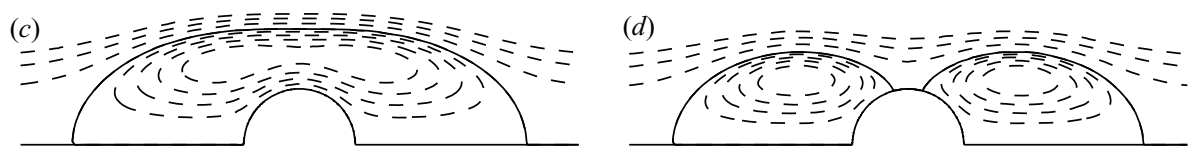

FIgURE 1. Examples of the four types of attached vortices $(\alpha=0)$ without swirl $(\lambda=0)$. Each is embedded in shear flow. The radius of the sphere is $a=1$ in each case. Each subplot shows streamlines in the meridional plane. Streamlines are dashed except for the vortex boundary which is solid. Flow parameters for the examples shown are: $(a)$ the trailing vortex, $\sigma=14, M=4 ;(b)$ the vortex band, $\sigma=0.5, M=0.66 ;(c)$ the surrounding vortex, $\sigma=0.5, M=12 ;(d)$ the symmetric pair of vortices behind and in front of the sphere, $\sigma=0.5$, $M($ total $)=7.5$.

the vortex cross-section in the meridional half-plane. There are likewise four nonswirling families for each value of $\sigma \geqslant 0$. The four families of attached vortices can be described as $(a)$ trailing vortex wakes, $(b)$ bands of vorticity around the sphere, $(c)$ vortices that surround the sphere and $(d)$ symmetric pairs of vortices fore and aft of the sphere. Figure 1 shows examples. The vortex cross-sections become increasingly elongated as the shear constant $\sigma$ increases.

There are attached vortices in family $(a)$ for all $M>0$ and $\sigma \geqslant 0$, but for the other families there are bound constaints on $M$, which depend on $\sigma$. For family $(c)$, given $\sigma$ there is a minimal value of $M$ for which we have found solutions, that minimal value increasing with $\sigma$. For $\sigma=0$ the minimal value of $M$ is 0 and for each $M$ the outer surface of the surrounding vortex is a concentric sphere; for $\sigma=0.5$ the minimal value of $M$ is approximately 6.6. As $M$ increases the outer boundary of the vortex becomes more elliptical. For families $(b)$ and $(d)$ there are maximal values of $M$, depending on $\sigma$, for which we have found solutions. For family $(b)$ that maximal value of $M$ decreases as $\sigma$ increases, while for family $(d)$ the maximal value of $M$ increases with $\sigma$. For $\sigma=0.5$ the maximal $M$ is about 0.66 for family $(b)$ and about 8.7 for family $(d)$. For $\sigma=0$ the corresponding values are about 1.55 and 4.65 respectively. For family $(d)$, as $M$ approaches the maximal value the attachment points in the meridional cross-section approach the top of the obstacle. The nature of these various bound constraints is consistent with the observation that the vortices become more elongated in the direction of the axis of symmetry as $\sigma$ increases.

There is a family of vortex rings, extending to all $\alpha<0$, for each attached vortex. One such family of trailing vortex rings with shear is shown in figure 2(a). Note that while the shear stretches out 'large' vortices, small-cross-section vortices are almost circular with or without shear. Figure $2(b)$ shows the effect on a small-cross-section vortex ring as shear increases while circulation and moment are held fixed: the radius of the ring decreases but there is little change in the position of the centre of the ring on the $z$-axis.

It can be noted in figure 1, that for trailing vortices embedded in flows with a large shear $(\sigma \gtrsim 7$ for $M=4)$, there is a stagnation bubble in front of the sphere. Such stagnation bubbles have been studied analytically by Rubel (1986) for shear flow past 
(a)

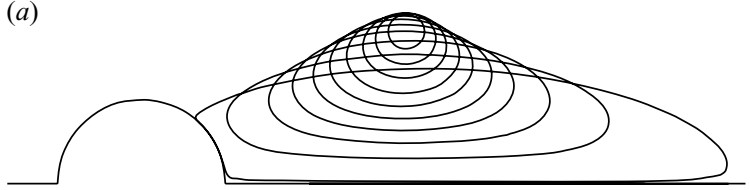

(b)

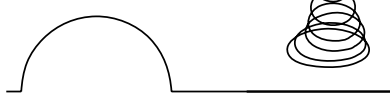

FiguRE 2. Two families of vortex rings with shear and no swirl. Vortex boundaries (only) are shown for several members of each family. (a) A family of trailing vortex rings with $\sigma=4$ and circulation $k=(\omega+\sigma) M=50$. The values of $\alpha$ are $-0.002,-0.31,-1.03,-2.05,-3.45$, $-5.33,-7.94,-11.6,-16.2,-24.18$. (b) A family of small-cross-section trailing vortex rings with $k=50$ and $M=0.25$. The values of the shear constant $\sigma$ for the rings shown (from top to bottom) are $0,1,2,4,8,14,22,30,40$ and 50 .

a sphere when there is no vortex. Such bubbles occur not only in the presence of attached vortices, but also for flows with vortex rings. For example, for the family of rings shown in figure $2(b)$, there is a stagnation bubble in front of the sphere for $\sigma>5$, the size of the bubble increasing with $\sigma$.

\subsection{Vortices with swirl}

For fixed $\alpha, M$ and $\sigma$ solutions can be found for all values of $\lambda, 0 \leqslant|\lambda| \leqslant$ some maximal value, where this maximal value depends on $\alpha, M$ and $\sigma$ and may also be different for each of the four families. (Note that the sign of $\lambda$ determines whether the flow swirls clockwise or counterclockwise, but the stream function depends only on $|\lambda|$.) As $|\lambda|$ increases, the value of $\omega$, which is determined as part of the solution, decreases. The maximal value of $|\lambda|$ is reached when $\omega+\sigma=0$. When $\omega+\sigma=0$, it follows from (1.4) that the velocity and vorticity vectors are parallel inside the vortex region. Such flows are called Beltrami flows. In other words, for fixed $\alpha(\leqslant 0), M$ and $\sigma$ there are one-parameter families of solutions, parametrized by either $\lambda$ or $\omega$, such that one end of each family is a Beltrami flow $(\omega+\sigma=0)$ and at the other end of the family is a non-swirling flow $(\lambda=0)$. Velocity and vorticity are parallel for Beltrami flow and perpendicular for non-swirling flow. Figure 3 compares two flows from one such family of trailing vortices, one with small swirl and the other near the Beltrami flow limit. In this figure, as is frequently, but not always, the case, the meridional-plane cross-sections for the flows in the same one-parameter family are nearly identical. The main distinction is in the relative pitch of the angle with which streamlines go around a toroidal surface $\psi=c$.

If there is no shear and $\alpha=0$, then there is a family of exact solutions with a sphere of radius $b$ as the outer vortex boundary. When there is no spherical obstacle such solutions were given by Moffatt (1969). If there is a spherical obstacle of radius $a$, the analysis in Moffatt (1969) shows that a solution to

$$
\mathrm{L} \psi=r^{2} \omega-\lambda^{2} \psi
$$

for $a<\rho<b$, where $\rho^{2}=r^{2}+z^{2}$, can be given explicitly in terms of Bessel functions of the first kind by

$$
\psi=r^{2}\left(\frac{\omega}{\lambda^{2}}+C_{1}(\lambda \rho)^{-3 / 2} J_{3 / 2}(\lambda \rho)+C_{2}(\lambda \rho)^{-3 / 2} J_{-3 / 2}(\lambda \rho)\right) .
$$


(a)
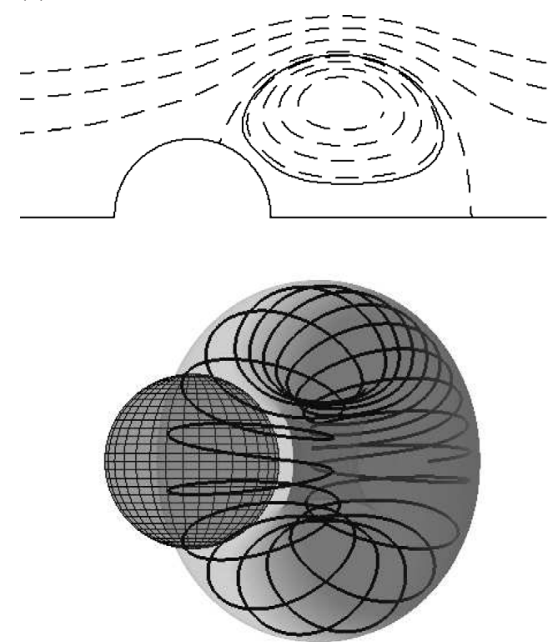

(b)
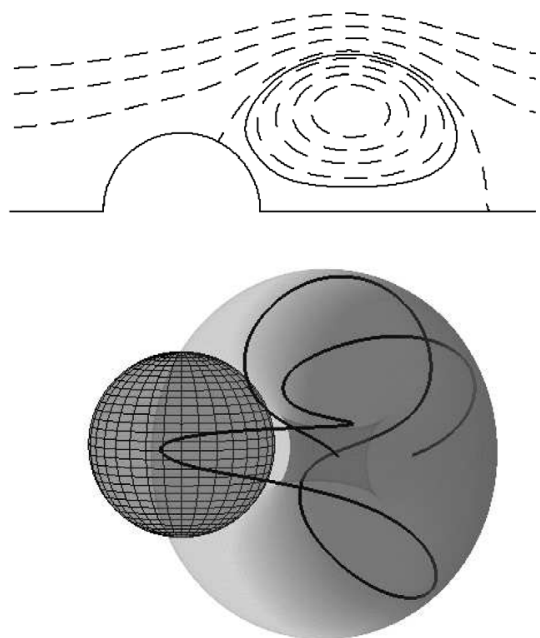

FIgURE 3. Two flows with for $\alpha=-0.15, \sigma=0$ and $M=4$, but with $\lambda^{2}=0.2$ in $(a)$ left and $\lambda^{2}=6$ in $(b)$. (The Beltrami flow for this family has $\lambda^{2}=6.25$ ). The meridional plane cross-sections of the flows are very similar, although not identical. In the three-dimensional plots the vortex core is shaded and part of a single interior streamline is shown. The streamline is shown for 20 revolutions around the toroidal surface for the ring in $(a)$, but for only 4 revolutions for the ring in $(b)$, showing the increase in the variation in $\theta$ per revolution for the flow with larger $|\lambda|$.

The function $\psi$ and the external flow $\psi_{e}=\frac{1}{2} r^{2}\left(1-b^{3} / \rho^{3}\right)$ must match at $\rho=b$ along with their normal derivatives. This, along with $\psi(a)=0$, leads to the nonlinear system

$$
\begin{aligned}
\frac{\omega}{\lambda^{2}}+C_{1}(\lambda a)^{-3 / 2} J_{3 / 2}(\lambda a)+C_{2}(\lambda a)^{-3 / 2} J_{-3 / 2}(\lambda a) & =0 \\
\frac{\omega}{\lambda^{2}}+C_{1}(\lambda b)^{-3 / 2} J_{3 / 2}(\lambda b)+C_{2}(\lambda b)^{-3 / 2} J_{-3 / 2}(\lambda b) & =0 \\
\frac{3}{2}(\lambda b)^{1 / 2}+C_{1} J_{5 / 2}(\lambda b)-C_{2} J_{-5 / 2}(\lambda b) & =0
\end{aligned}
$$

for $C_{1}, C_{2}$ and $b$ given $a, \lambda$ and $\omega$. When this system is solvable there is a spherical vortex.

Generally, when there is a spherical obstacle the four classes of vortices, $(a)-(d)$, described above also exist for flows with swirl. In fact, for Beltrami flows there are two families of vortices surrounding the sphere, the spherical vortices and also a non-spherical family. Figure 4 shows an example where both vortices have the same input parameters $\alpha=0, \omega=\sigma=0$ and $M=14$. The two families appear to merge at about $M=18$. For $M<13$ solutions in the second family split into two symmetric regions of vorticity fore and aft of the sphere. Solutions in the second family can be continued to somewhat smaller $\lambda$ (and correspondingly, $\omega$ somewhat larger than 0 ), but we have not been able to continue that family all the way to $\lambda=0$.

\subsection{Rings when there is no obstacle}

Rings with swirl, but no shear, were computed previously in Eydeland \& Turkington (1988), using a numerical algorithm based on the variational principle described in Turkington (1989). As they note, there is a two-parameter family of rings with swirl 
(a)
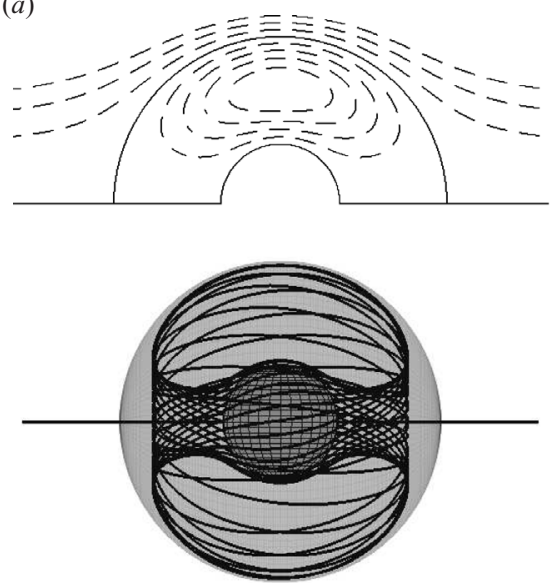

(b)
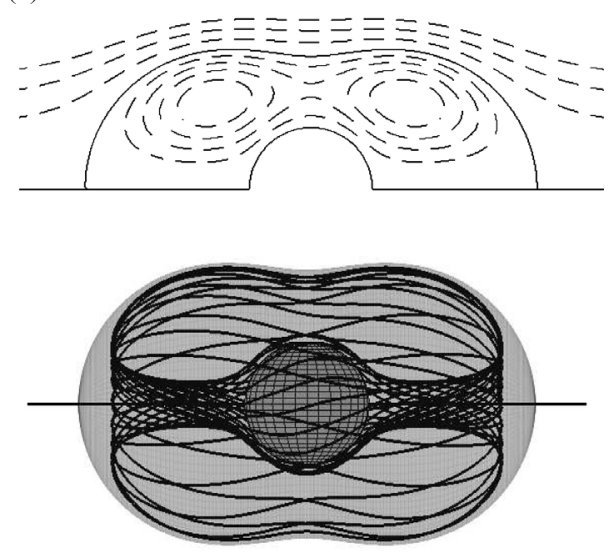

Figure 4. Two attached Beltrami flows surrounding the unit sphere with $\sigma=0$ and $M=14$. The values of $\lambda^{2}$ are 3.64 for the spherical solution $(a)$ and 4.31 for the second solution $(b)$. On both plots a single streamline is shown for 30 revolutions around the toroidal surface for the stream function value -0.5 .

(a)

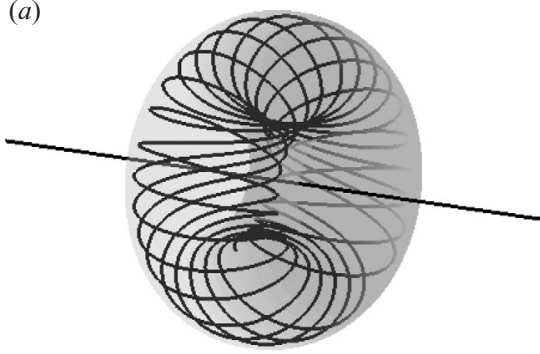

(b)

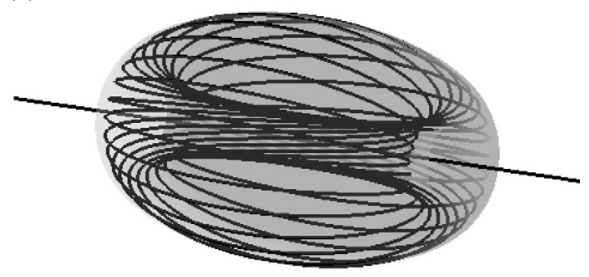

FIgURE 5. Two rings with swirl; one embedded in shear flow. Normalized with $M=1$, both flows are for the parameters $\alpha=-0.1, \lambda=3$. The values of $\sigma$ are 0 in $(a)$ and 16 in $(b)$.

and no shear. Figure 5 compares a vortex ring in shear flow with a ring in a flow with no shear.

In general, when there is no obstacle, by a similarity transformation one of the non-zero parameters we are using may be set to 1 . In particular, if $\tilde{\psi}(\tilde{r}, \tilde{z})$ is a solution for the parameters $\tilde{\omega}, \tilde{\lambda}, \tilde{M}, \tilde{\alpha}$ and $\tilde{\sigma}$, then defining $\psi(r, z)=b^{-2} \tilde{\psi}(\tilde{r}, \tilde{z})$ where $\tilde{r}=b r$ and $\tilde{z}=b z$, gives a solution, also with velocity unity at infinity, for parameters $\omega=b^{2} \tilde{\omega}, \lambda=b \tilde{\lambda}, M=b^{-3} \tilde{M}, \alpha=b^{-2} \tilde{\alpha}$ and $\sigma=b^{2} \tilde{\sigma}$. Therefore, instead of four independent parameters there are now three. Without the obstacle we have found at most one solution for each set of parameters. This is consistent with the uniqueness result proved in Amick \& Fraenkel (1988) for vortex rings without swirl or shear.

In the final section of the paper it is shown that if there is shear but no swirl, then there is an analogue of Hill's spherical vortex in which the boundary is a prolate spheroid. Figure $6(a)$ shows an example with $M=5$ and $\sigma=9$. Figure $6(b)$ shows the attached Beltrami flow with the same $M$ and $\sigma$. For that flow the meridional plane cross-section is clearly not an ellipse. 
(a)
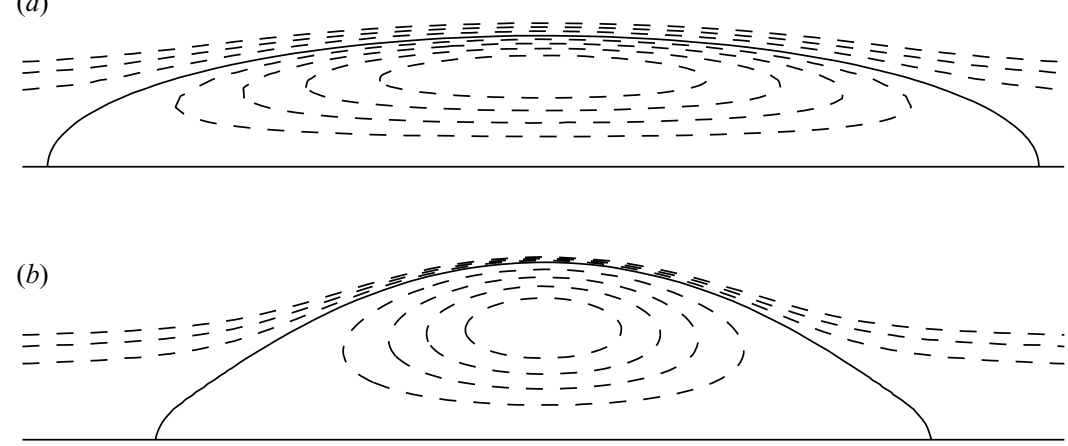

Figure 6. The non-swirling $(a)$ and Beltrami $(b)$ attached vortices for $M=5, \sigma=9$. For $(a)$, the meridional-plane cross-section of the vortex boundary is an ellipse.

\subsection{Vortex tubes}

Each attached vortex (except for the vortex bands, figure 1(b), which do not extend to the axis of rotation), can be perturbed to a family of solutions of (2.2), with $\alpha>0$ and having the same values of the parameters $\omega, \lambda$ and $\sigma$ as the attached vortex. The vortex support of these solutions extends to infinity along the axis of symmetry and we refer to them as vortex tubes. The moment $M$ of the vortex support in the full physical space is not defined, so we do not use $M$ as a parameter in discussing the vortex tube solutions. However, we do use the moment of the truncated domain $\left\{(z, r) \in D_{n}: z^{2}+r^{2}<R^{2}\right\}$, where $R$ is the truncation radius as described earlier, in order to stabilize the numerical method as before. Now there is an additional iteration level needed to vary $M$ so as to achieve the desired values of both $\omega$ and $\lambda$.

If $\lambda \neq 0$, then for any streamline value $c, 0<c<\alpha$, the corresponding streamlines will have a helical shape, distorted by the sphere and the re-circulating core of the vortex. One such streamline is plotted in each three-dimensional plot of a vortex tube. For $c<0$, the streamlines stay in a bounded wake region; for $c>\alpha$ each streamline is non-helical, staying in a single constant- $\theta$-plane.

Three vortex tubes, all with the same values of $\omega, \lambda, \sigma$ and $\alpha$, are shown in figure 7 . The first two are perturbations of a symmetric attached vortex and a trailing attached vortex, respectively. The third is obtained by perturbation from the background irrotational or uniform shear flow. Figure 8 shows tubes in the same three families, but for a larger value of $\alpha$. It can be noted by comparing figures 7 and 8 , that as $\alpha$ increases the solutions in the first two families get flatter and more stretched out, while for the perturbations of background flow the vortex boundary becomes higher and develops a thin wake that grows as $\alpha$ increases. There is a value of $\alpha$, the maximum value for which solutions exist, at which all three families come together. This will be discussed further at the end of this section. Figure 9 shows a solution for $\alpha$ near the maximal value. The recirculation region extends beyond the margins of the plot.

There are two families of vortex tubes when there is no obstacle: perturbations of attached vortices and perturbations from the background irrotational or uniform shear flow. Two examples of perturbations of attached vortices are given in figure 10 . Figure 10 highlights the difference in the $\psi=0$ streamline profiles for a flow without swirl compared with one in which $\lambda$ is large. For flows with swirl there is a thin elongation of the recirculation region that becomes wider and longer as $\alpha$ increases. 
(a)

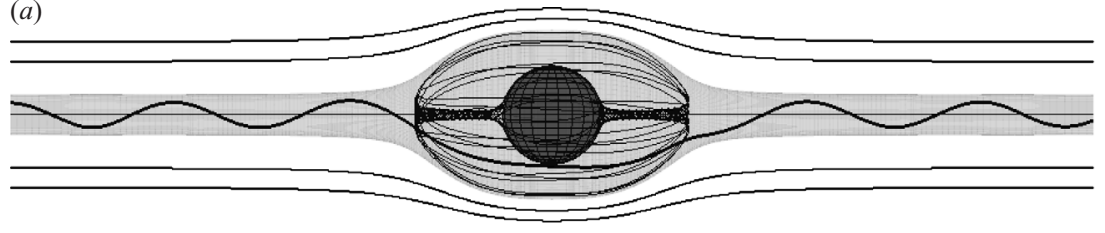

(b)

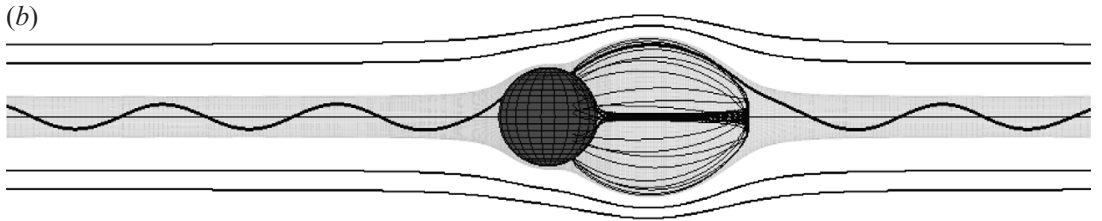

(c)

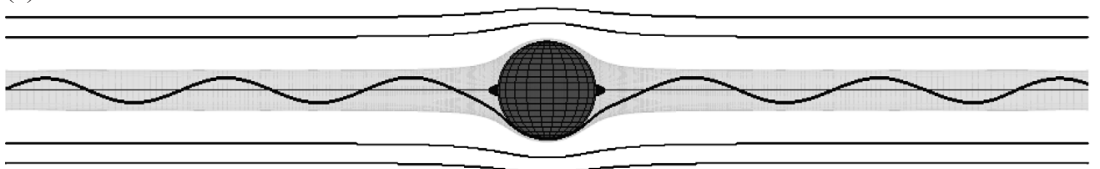

Figure 7. Three vortex tubes for $\omega=2, \lambda^{2}=1.5, \sigma=0$ and $\alpha=0.075$. Tube $(a)$ is obtained by continuation from a symmetric, annular attached vortex with the same $\omega, \sigma$ and $\lambda$; $(b)$ by continuation from a trailing vortex; $(c)$ is a perturbation of the background irrotational flow. In each plot two streamlines in the vortex region are shown: a helical streamline $\psi=c$, for some $c, 0<c<\alpha$, and 20 loops of a single steamline in the wake region, $\psi<0$.

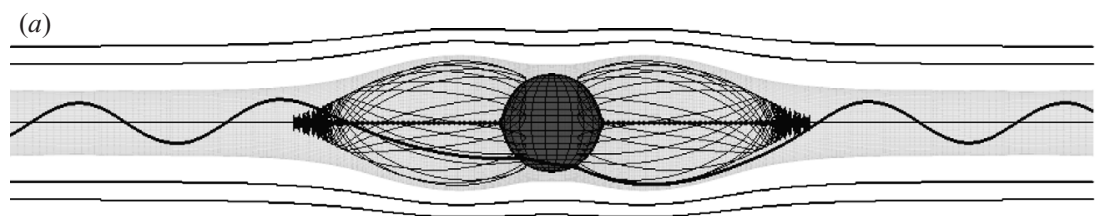

(b)

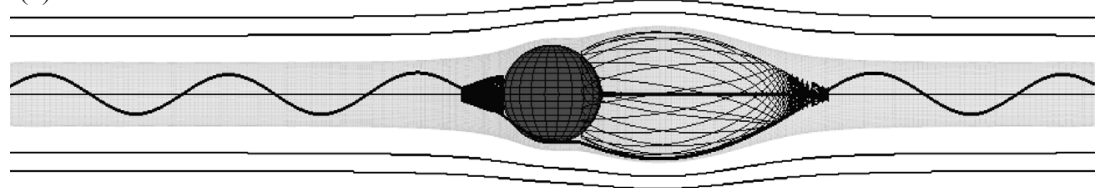

(c)

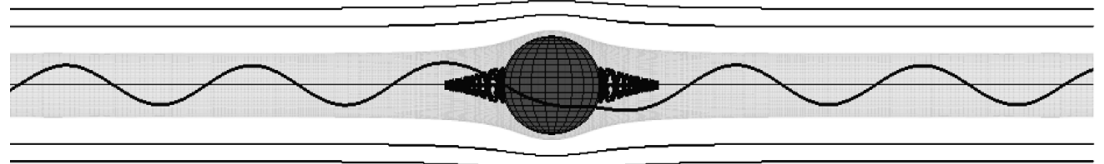

FiguRE 8. As Figure 7 except with $\alpha=0.15$. As $\alpha$ increases vortex boundaries in $(a)$ and $(b)$ become lower near the recirculation region, while solutions in $(c)$ become higher. The three families merge at a solution with maximal $\alpha$, which is shown in figure 9 . 
(a)

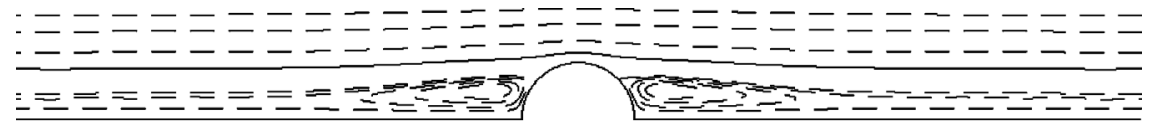

(b)

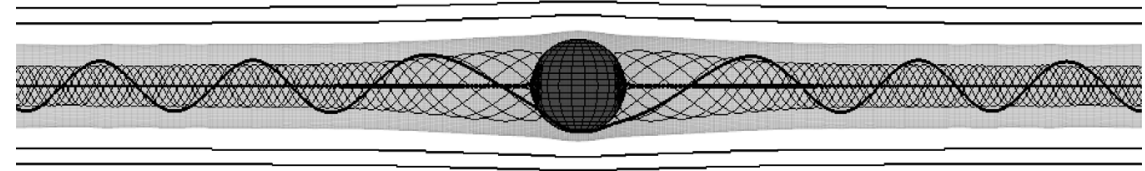

FIGURE 9. Near the maximum- $\alpha$ solution for the families in figures 7 and 8 . The value of $\alpha$ is slightly less than 0.2 . Two streamlines are shown in $(b)$. The helical streamline $\psi=c_{1}>0$, is printed somewhat thicker. The streamline for $\psi=c_{2}<0$ (shown for 9 loops) follows the helical streamline closely until a point well beyond the margins of the plot where it is pulled into the inner part of the toroidal surface $\psi=c_{2}$. That inner part of the surface appears in the plot as a thickening of the axis of symmetry. See figure 11 for a detail in a comparable situation.
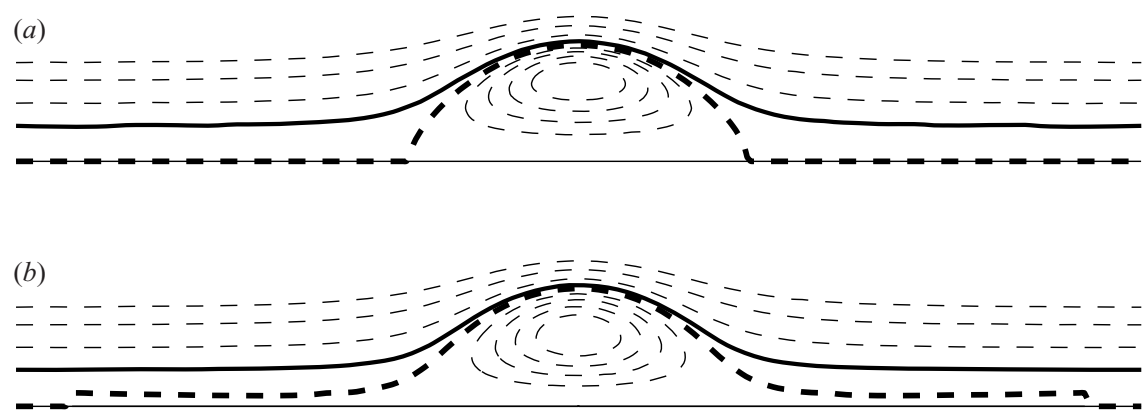

FIGURE 10. Streamlines are shown for two vortex tubes, $(a)$ without swirl, $(b)$ a Beltrami flow $\left(\lambda^{2}=3.5\right)$, with $\alpha=0.17$ in both cases. The $\psi=0$ streamline is highlighted. The narrow elongation of the recirculation wake in $(b)$ is typical for tubular flows with swirl.

This elongation is somewhat noticable in the three subplots of figure $8(a-c)$, is very prominent in figure 10, and for the maximum- $\alpha$ solution shown in figure 9 it dominates the flow. Figure 11 shows a blow-up of two streamlines near the point where the 0 streamline in figure $10(b)$ intersects the axis. Physically, the swirl around the axis in the tubular region forces the recirculation region to become more extended.

For flows with swirl and with $\alpha$ near the maximum value, the computed attachment points of the $\psi=0$ streamline on the axis of symmetry are very near $\pm R$, so are dependent on the chosen grid and probably not computed very accurately. Nevertheless it is apparent from figure 9 that as the radius of the sphere goes to zero, these maximal solutions approach a solution independent of $z$. Indeed, when there is no obstacle the perturbations of irrotational flow have stream functions that are independent of $z$ which can be analysed analytically. This analysis will give some mathematical insight not only into why the zero streamline becomes elongated when there is swirl, but also into why there is a maximum $\alpha$ for which solutions exist.

We carry out the details of this analysis only in the case of Beltrami flow without shear, $\omega=\sigma=0$; the more general case is similar but more involved. We look for 

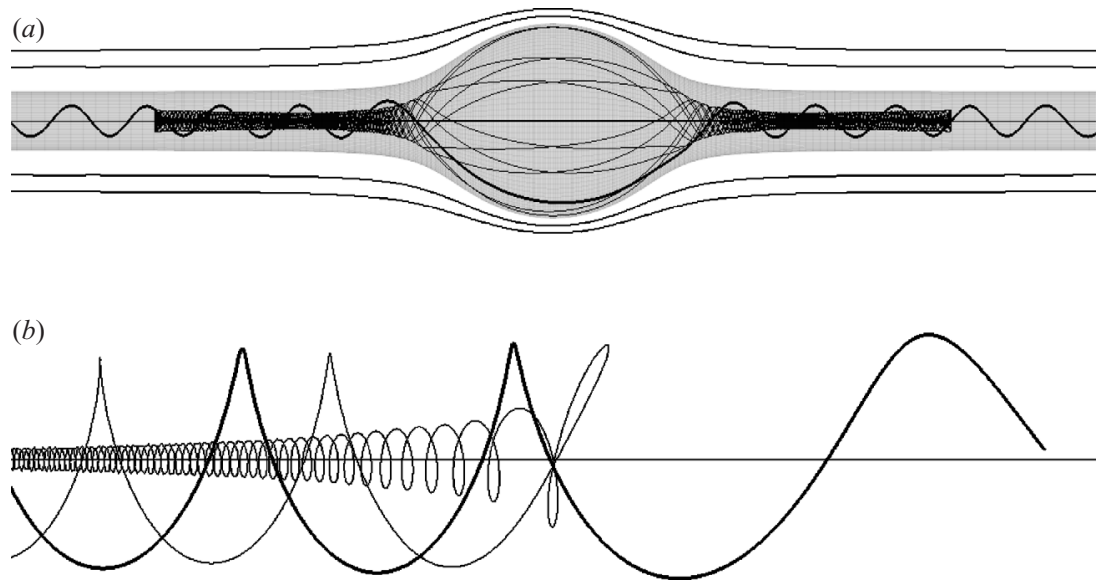

Figure 11. (a) The three-dimensional view of the Beltrami flow in figure 10. (b) A close-up view of two streamlines near the point where the 0 streamline intersects the axis. The streamline that maintains its helical shape has streamline value slightly greater than 0 . The other streamline, with streamline value $c<0$, is in the recirculation region and as it flows from right to left spirals very tightly around the axis on the inner side of the toroidal surface $\psi=c$.

solutions to

$$
\begin{aligned}
\psi^{\prime \prime}(r)-\frac{1}{r} \psi^{\prime}(r)+\lambda^{2} \psi(r) & =\lambda^{2} \alpha, \quad \psi<\alpha, \\
\psi^{\prime \prime}(r)-\frac{1}{r} \psi^{\prime}(r) \quad & =0, \quad \psi>\alpha .
\end{aligned}
$$

The first equation transforms to Bessel's equation of order 1 and the solution is

$$
\psi= \begin{cases}\alpha+C_{1} y_{1}+C_{2} y_{2}, & r<r_{o}, \\ \frac{1}{2}\left(r^{2}-r_{o}^{2}\right)+\alpha, & r>r_{o},\end{cases}
$$

where $y_{1}=r J_{1}(\lambda r), y_{2}=r Y_{1}(\lambda r)$ and $C_{1}, C_{2}$ and $r_{o}$ are chosen so that

$$
\left.\begin{array}{l}
C_{1} y_{1}+C_{2} y_{2}=0, \\
C_{1} y_{1}^{\prime}+C_{2} y_{2}^{\prime}=r_{o},
\end{array}\right\}
$$

and $\psi(0)=0$. Since

$$
s Y_{1}(s) \rightarrow-2 / \pi \text { as } s \rightarrow 0,
$$

$\psi(0)=0$ will be satisfied by taking $C_{2}=\lambda \alpha \pi / 2$. By Abel's identity the Wronskian $W=W\left(y_{1}, y_{2}\right)=c r$ and using (3.3), again, $c=2 / \pi$. Solving (3.2) gives

$$
C_{2}=\frac{r_{o} y_{1}\left(r_{o}\right)}{W\left(r_{o}\right)}=\frac{\pi}{2} r_{o} J_{1}\left(\lambda r_{o}\right),
$$

so $r_{o}$ is determined by

$$
\lambda r_{o} J_{1}\left(\lambda r_{o}\right)=\lambda^{2} \alpha
$$

Note that the velocity on the axis:

$$
\lim _{r \rightarrow 0} \psi^{\prime}(r) / r=\lim _{r \rightarrow 0} \lambda\left(C_{1} J_{0}(\lambda r)+C_{2} Y_{0}(\lambda r)\right)=-\infty .
$$


Since $\psi^{\prime}\left(r_{o}\right)>0$, there is a flow reversal in the region of vorticity. Flows that we are considering have a single flow reversal, i.e. there is a single positive root of $C_{1} J_{0}(\lambda r)+C_{2} Y_{0}(\lambda r)$ to the left of $r_{o}$. Equation (3.4) has solutions $r_{o}$ for all $\alpha$, but if the first $r_{o}>0$ solving (3.4) is to the right of the first relative maximum of $r J_{1}(\lambda r)$, then there are two positive roots of $J_{1}(\lambda r)$ to the left of $r_{o}$, and hence two positive roots of $C_{1} J_{0}(\lambda r)+C_{2} Y_{0}(\lambda r)$ to the left of $r_{o}$. Since the first relative maximum of $J_{1}$ occurs at the first positive root $j_{0}$ of $J_{0}$, it follows that the maximum possible $\alpha$ is

$$
\alpha_{\max }=j_{0} J_{1}\left(j_{0}\right) / \lambda^{2} \text {. }
$$

For $\lambda^{2}=2.9$ and $\omega=\sigma=0$ and with a spherical obstacle of radius 1 , the maximum value of $\alpha$ for which we could obtain solutions is $\alpha=0.426$. For $\lambda^{2}=2.9$ formula (3.5) gives $\alpha_{\max }=0.4305$. Also the value of $r$ in the far field on the upper boundary of the vortex region for the computed solution is 1.42 , comparable to the value of $r_{o}=1.41$ obtained from (3.4). This numerical comparison shows, as expected, that the presence of an obstacle does not greatly effect the flow in the far field, and also provides some indication that our method is reasonably accurate even in this extreme case.

\section{Spheroidal vortex in flows with shear}

It will be shown in this section that there is an analogue of Hill's spherical vortex for axisymmetric shear flow (without swirl) in which the vortex boundary is a prolate spheroid. An explicit formula is given in Rubel (1986) for the stream function $\psi_{e}$ for shear flow exterior to a spheroid, with $\psi_{e}=0$ on the surface of the spheroid. The stream function $\psi_{i}$ for vortex flow on the interior of the spheroid $z^{2} / a^{2}+r^{2} / b^{2}=1$ can be given by

$$
\psi_{i}=H r^{2}\left(1-\frac{z^{2}}{a^{2}}-\frac{r^{2}}{b^{2}}\right),
$$

which satisfies $\mathrm{L} \psi_{i}=\omega r^{2}$ if

$$
2 H=\frac{-\omega a^{2} b^{2}}{\left(4 a^{2}+b^{2}\right)} .
$$

It is to be shown that, given $\omega$ and the shear parameter $\sigma$, the normal derivatives $\partial \psi_{e} / \partial n$ and $\partial \psi_{i} / \partial n$ agree on the boundary of some prolate spheroid. For this it is convenient to use elliptical coordinates $\mu$ and $\zeta$ (Lamb 1932, p. 142), where for a prolate ellipse,

$$
z=k \mu \zeta, r=k \sqrt{1-\mu^{2}} \sqrt{\zeta^{2}-1}
$$

For constant $\zeta=\zeta_{0}>1$, as $\mu$ varies from -1 to $1, z$ and $r$ vary over the upper half of the ellipse $z^{2} / a^{2}+r^{2} / b^{2}=1$ for $a=k \zeta_{0}$ and $b=k \sqrt{\zeta_{0}^{2}-1}$. The focal length and eccentricity of the ellipse are $2 k$ and $e=1 / \zeta_{0}$ respectively. The $\mu, \zeta$ coordinate system is orthogonal, so requiring $\partial \psi_{e} /\left.\partial \zeta\right|_{\zeta=\zeta_{0}}=\partial \psi_{i} /\left.\partial \zeta\right|_{\zeta=\zeta_{0}}$ is equivalent to matching normal derivatives on the boundary of the spheroid. Substituting (4.3) into (4.1), using (4.2) and differentiating gives

$$
\partial \psi_{i} /\left.\partial \zeta\right|_{\zeta=\zeta_{0}}=k^{4} \omega \zeta_{0}\left(1-\mu^{2}\right)\left(\zeta_{0}^{2}-\mu^{2}\right)\left(\zeta_{0}^{2}-1\right) /\left(5 \zeta_{0}^{2}-1\right)
$$

In particular, as a function of $\mu, \partial \psi_{i} /\left.\partial \zeta\right|_{\zeta=\zeta_{0}}$ has the form $\left(1-\mu^{2}\right)\left(A_{i}+B_{i} \mu^{2}\right)$ where the coefficients $A_{i}$ and $B_{i}$ depend on $k, \omega$ and $\zeta_{0}$. It follows by differentiating equation (16) of Rubel (1986) that $\partial \psi_{e} /\left.\partial \zeta\right|_{\zeta=\zeta_{0}}$ likewise has the form $\left(1-\mu^{2}\right)\left(A_{e}+B_{e} \mu^{2}\right)$ with 
coefficients $A_{e}$ and $B_{e}$ depending on $k, \sigma$ and $\zeta_{0}$. Therefore, normal derivatives match on the boundary if $A_{i}=A_{e}$ and $B_{i}=B_{e}$, giving two equations which, for given $\omega$ and $\sigma$, can be solved for $k$ and $\zeta_{0}$. To be specific, using equation (16) of Rubel (1986) and letting $Q_{m}$ denote the Legendre function of the second kind of order $m$, the equations to be solved can be reduced to

$$
\begin{gathered}
2 \zeta_{0}-\frac{Q_{3}^{\prime \prime}\left(\zeta_{0}\right)}{Q_{3}^{\prime}\left(\zeta_{0}\right)}\left(\zeta_{0}^{2}-1\right)-\frac{8 \omega}{\sigma} \frac{\zeta_{0}}{5 \zeta_{0}^{2}-1}=0 \\
\frac{1}{4} \sigma\left(\zeta_{0}^{2}-1\right)\left(\frac{2 \zeta_{0}}{\zeta_{0}^{2}-1}-\frac{1}{5} \frac{Q_{3}^{\prime \prime}\left(\zeta_{0}\right)}{Q_{3}^{\prime}\left(\zeta_{0}\right)}-\frac{4}{5} \frac{Q_{1}^{\prime \prime}\left(\zeta_{0}\right)}{Q_{1}^{\prime}\left(\zeta_{0}\right)}\right)-2 \omega \frac{\zeta_{0}^{3}}{5 \zeta_{0}^{2}-1}=\frac{1}{k^{2}} \frac{Q_{1}^{\prime \prime}\left(\zeta_{0}\right)}{Q_{1}^{\prime}\left(\zeta_{0}\right)}
\end{gathered}
$$

Thus the focal length and eccentricity of a prolate spheroid can be determined so that the corresponding exterior and interior flows match on the boundary.

Spheroidal vortex boundaries determined by solving these equations match quite closely those found by our general numerical procedure. In a test case with $\sigma=1$ and $\omega=6.17$ and using a 190 by 402 grid, the normal distance between the computed vortex boundary and the elliptical boundary was less than 0.002 , i.e. to about visual accuracy for an ellipse with major and minor axes of lengths 3.26 and 2.35. In this case the transplanted grid spacing in the physical domain near the ellipse is about $h=0.02$, so the error in the computation of the vortex boundary is about one order of magnitude smaller than the grid spacing.

Note that figure 6 shows that when swirl is added, the analogues of the Moffatt vortices in shear flow do not have elliptical cross-sections.

\section{Conclusions}

We have developed a general procedure for finding axisymmetric vortices with swirl which are steady with respect to a background flow for which the far-field velocity is $v_{z} \sim\left(1+\frac{1}{2} \sigma r^{2}\right), \sigma \geqslant 0$ a shear parameter. There may, or may not, be a spherical obstacle in the flow. Flows are found by numerically solving the Bragg-Hawthorne equation using a non-Newton-based iterative procedure.

In addition to vortex rings with swirl, we have found tubular vortices extending to infinity, as well as vortices attached to the spherical obstacle, and when there is no obstacle, analogues of the Hill and Moffatt spherical vortices. When there is a spherical obstacle we have generally found multiple solutions for each set of parameters, as seen for example, in figures $1,4,7$ and 8 .

The general effect of shear is to flatten and stretch out large-cross-section vortices. Small-cross-section vortex rings have basically circular cross-sections, but increasing shear decreases the radius of the vortex ring (see figure 2). For trailing vortices, if the shear is sufficiently large, a stagnation bubble forms in front of the sphere. We have shown analytically that the analogue of Hill's spherical vortex in the presence of uniform shear, with no swirl and no obstacle, has an elliptical cross-section.

We have found families of vortices with swirl connecting each non-swirling vortex flow to a Beltrami flow (i.e. connecting a flow in which vorticity and velocity are perpendicular to one in which vorticity and velocity are parallel). In each case, the swirl parameter cannot be increased beyond the value which corresponds to Beltrami flow. For vortex rings, as the swirl parameter increases, the cross-sections in the meridional plane only vary slightly although the flow pattern inside the stream surface bounding the vortex varies considerably. However, for vortex tubes there is more distinct difference between the cross-sections for non-swirling flows and flows with large swirl, as shown in figures 10 and 11. The swirling flow around the axis 
stretches out the ends of the recirculating flow region. As for non-swirling flow, there is a maximum value of $\alpha$ for which steady swirling flows exist.

\section{REFERENCES}

Amick, C. J. \& Fraenkel, L. E. 1988 The uniqueness of a family of steady vortex rings. Rat. Mech. Anal. 100, 207-241.

BAtChelor, G. K. $1956 a$ On steady laminar flow with closed streamlines at large Reynolds number. J. Fluid Mech. 1, 177-190.

BATCHELOR, G. K. 1956b A proposal concerning laminar wakes behind bluff bodies at large Reynolds numbers. J. Fluid Mech. 1, 388-398.

Batchelor, G. K. 1967 An Introduction to Fluid Dynamics. Cambridge University Press.

Elcrat, A., Fornberg, B., Horn, M. \& Miller, K. 2000 Some steady vortex flows past a circular cylinder. J. Fluid Mech. 409, 13-27.

Elcrat, A., Fornberg, B. \& Miller, K. 2001 Some steady axisymmetric vortex flows past a sphere. J. Fluid Mech. 433, 315-328.

Elcrat, A. R. \& Miller, K. G. 2003 A monotone iteration for axisymmetric vortices with swirl. Diff Integral Equat. 16, 949-968.

Eydeland, A. \& TuRKIngton, B. 1988 A numerical study of vortex rings with swirl. GANG Report 12, Series I, University of Massachussetts, Amherst.

ForNBERG, B. 1993 Computing steady incompressible flows past blunt bodies- A historical overview. Numerical Methods for Fluid Dynamics, (ed. W. Baines \& R. Morton). Oxford University Press.

Funumoto, Y. 2002 Higher-order asymptotic theory for the velocity field induced by an inviscid vortex ring. Fluid Dyn. Res. 30, 65-92.

Hicks, W. 1899 Research in vortex motion. III. On spiral or gyrostatic vortex aggregates. Phil. Trans. R. Soc. Lond. A 176, 33-101.

Lamb, H. 1932 Hydrodynamics, 6th ed. Cambridge University Press.

Lifschitz, A., Suters, W. H. \& Beale, J. T. 1996 The onset of instability in exact vortex rings with swirl. J. Comput. Phys. 129, 8-29.

Linden, P. F. \& TuRner, J. S. 2001 The formation of 'optimal' vortex rings, and the efficiency of propulsion devices. J. Fluid Mech. 427, 61-72.

Moffatt, H. K. 1969 The degree of knottedness of tangled vortex lines. J. Fluid Mech. 35, 117-129.

Mohseni, K. \& Gharib, M. 1998 A model for universal time scale of vortex ring formation. Phys. Fluids 10, 2436-2438.

Norbury, J. 1973 A family of steady vortex rings. J. Fluid Mech. 57, 417-431.

Rubel, A. 1986 Axisymmetric shear flow over spheres and spheroids. AIAA J. 24, 630-634.

Turkington, B. 1989 Vortex rings with swirl: axisymmetric solutions of the Euler equations with nonzero helicity. SIAM J. Math Anal. 20, 57-73.

WAKelin, S. L. \& Riley, N. 1997 On the formation and propagation of vortex rings and pairs of vortex rings. J. Fluid Mech. 332, 121-139.

Wu, J.-Z., MA, H.-Y. \& ZHou, M.-D. 2006 Vorticity and Vortex Dynamics. Springer. 